Review

\title{
Eighteen Years of Molecular Genotyping the Hemophilia Inversion Hotspot: From Southern Blot to Inverse Shifting-PCR
}

\author{
Liliana C. Rossetti *, Claudia P. Radic, Miguel M. Abelleyro, Irene B. Larripa and Carlos D. De Brasi \\ Departamento de Genética, Instituto de Investigaciones Hematológicas, Academia Nacional de \\ Medicina, Pacheco de Melo 3081, Ciudad de Buenos Aires (CP 1425), Argentina; \\ E-Mails: pradic@ hematologia.anm.edu.ar (C.P.R.); mmabelleyro@ hematologia.anm.edu.ar (M.M.A.); \\ ibl@ hematologia.anm.edu.ar (I.B.L.); cdebrasi@hematologia.anm.edu.ar (C.D.D.B.)
}

* Author to whom correspondence should be addressed; E-Mail: rossetti @ hematologia.anm.edu.ar; Tel.: +5411-4805-5759; Fax: +5411-4803-9475.

Received: 5 September 2011; in revised form: 8 October 2011 / Accepted: 19 October 2011 / Published: 24 October 2011

\begin{abstract}
The factor VIII gene (F8) intron 22 inversion (Inv22) is a paradigmatic duplicon-mediated rearrangement, found in about one half of patients with severe hemophilia A worldwide. The identification of this prevalent cause of hemophilia was delayed for nine years after the $F 8$ characterization in 1984. The aim of this review is to present the wide diversity of practical approaches that have been developed for genotyping the Inv22 (and related int $22 h$ rearrangements) since discovery in 1993. The sequenceSouthern blot, long distance-PCR and inverse shifting-PCR - for Inv22 genotyping is an interesting example of scientific ingenuity and evolution in order to resolve challenging molecular diagnostic problems.
\end{abstract}

Keywords: F8; HEMA; intron 22 inversions; IS-PCR; LD-PCR

\section{Introduction}

Scientific development is not smoothly continuous but rather occurs in steps. There are several examples that prove the causative connection between each one of these steps and the use of novel experimental approaches. A typical example in the area of life sciences is the method of polymerase chain reaction (PCR) [1], which has revolutionized molecular diagnosis in medicine. Therefore, to tell 
the story of technical developments in a scientific discipline is perhaps the best way to understand it in depth. Notably, due to the molecular characteristics of the genes involved in hemophilia A and B (i.e., their different molecular sizes and structure complexities) a significant number of scientists who designed and developed innovative technical approaches for mutation detection and genotyping, worked in hemophilia.

Hemophilia A (HA) (OMIM 306700) is the most severe inherited bleeding disorder that affects humans. A deficiency in FVIII clotting activity leads to this coagulopathy, which affects 1 in 5,000 males worldwide. This makes HA one of the most common X-linked inherited diseases. Virtually all patients with HA associate with deleterious mutations within the coagulation factor VIII gene (F8). A familial history of the disease is known in about two thirds of cases, and it appears sporadically in one third of cases. HA is expressed in a wide range of clinical severities and these differences associate with the type and location of the causative gene defect. Therefore, HA is caused by a heterogeneous spectrum of molecular defects in $F 8$ including deletions, large DNA inversions, nonsense mutations, ins/del-frameshifts, splice variants and a large number of missense point mutations, all of which can cause defects in the expression, secretion, and/or half-life of FVIII in circulation.

HA can be classified by the residual clotting activity of FVIII as severe, moderate or mild disease, affecting about $40 \%, 10 \%$ and $50 \%$ of patients with HA, respectively. As a recessive X-linked disorder, the residual activity of plasmatic FVIII in heterozygous carrier females of severe $F 8$ mutations is usually $\sim 50 \%$ with respect to a non-carrier individual. Although extremely rare, homozygous females may also suffer from hemophilia in a similar way to hemizygous male patients [2]. However, most of the few cases of hemophilia expression in females are due to the coexistence of skewed Lyonization (biased Xchromosome inactivation) and the heterozygous carrier condition [3].

An international database, the HA mutation, structure, test and resource site (HAMSTeRS, URL: http://hadb.org.uk) contains extensive information, including a curated list of previously reported mutations and polymorphisms in F8 [4]. Today, 1,209 total unique mutations of different types are collected in the worldwide database HAMSTeRS, and 797 are single-base substitutions (point mutations) (database accessed 17/10/2011). Approximately one half of the severe cases of HA are caused by inversions between a sequence located within intron 22 of the $F 8$ gene and sequences outside the F8 gene.

Also characteristic of HA is the development of inhibitory antibodies against therapeutic FVIII (inhibitors) in approximately $15-35 \%$ of patients with severe HA. Particularly, FVIII inhibitors neutralize the substituted FVIII in about $21 \%$ of intron 22 inversions (a large series of patients with severe HA from the Bonn Centre, Germany) [5], a rate slightly higher than the average across all severe HA causative mutations, but lower than those cases associated with large deletions or nonsense mutations.

\section{Milestones in Hemophilia A Mutation Characterization}

\subsection{4-1993: Cloning and Characterization of the Human Coagulation Factor VIII}

The human $F 8$ gene was cloned between 1982 and 1984 [6]. At that time the gene was the largest described [6], and at approximately $187 \mathrm{~kb}$, remains one of the largest (chrX:154,064,070-154,250,998, UCSC genome browser, access date 17/10/2011 [7]). Genetic mapping positioned the $F 8$ gene in the 
most distal band (Xq28) of the long arm of the X-chromosome. The $F 8$ gene contains 26 exons, which vary in length from 69 to 3,106 base pairs (bp). Intron sequences correspond to $177.9 \mathrm{~kb}$, and are removed from the primary transcript product during splicing to generate a mature $F 8 \mathrm{mRNA}$ of approximately $9 \mathrm{~kb}$ in length that predicts a precursor protein of 2,351 amino acids. Of the larger intron sequences, we found six that are greater than $14 \mathrm{~kb}$ (introns 1, 6, 13, 14, 22 and 25), with intron 22 the largest at $32.8 \mathrm{~kb}$ in length [6].

Levinson et al. (1990) [8] found a curious example of a gene within a gene. Looking for transcripts within a region of Xq28 associated with several neurological disorders, the authors identified a CpG island in the largest $F 8$ intron. This $\mathrm{CpG}$ island was associated with a $1.8 \mathrm{~kb}$ transcript referred to as the A gene $(F 8 A)$. The nested $F 8 A$ gene was oriented in opposite direction to that of $F 8$ and contained no intervening sequences. Computer analysis of the sequence suggested that the $F 8 A$ gene encodes a protein with the complication that codon usage analysis suggested a frameshift halfway through the gene. Freije and Schlessinger (1992) [9] subsequently demonstrated that the X-chromosome contains three copies of $F 8 A$ and its adjacent regions, one in intron 22 and two telomeric and approximately $500 \mathrm{~kb}$ upstream to the $F 8$ gene transcription start site.

In 1992, Levinson et al. reported another transcript of $2.5 \mathrm{~kb}, F 8 B$, that emanates from the same $F 8$ intron $22 \mathrm{CpG}$ island as $F 8 A$ and transcribes in the same direction as $F 8$. The divergent transcripts $F 8 A$ and $F 8 B$ originate from within 122 bases of each start point. The newly identified 5' exon of $F 8 B$ in $F 8$ intron 22 potentially codes for eight amino acids and was spliced to $F 8$ exons 23-26, with the $F 8$ reading frame maintained [10].

Following these discoveries, Lakich et al. (1993) [11] pointed out that intron 22 was unusual in many respects. Containing $32.8 \mathrm{~kb}$, it is the largest intron in the $F 8$ gene. It also contains a CpG island, located about $10 \mathrm{~kb}$ downstream of exon 22 [11]. This $\mathrm{CpG}$ island appears to serve as a bidirectional promoter for the $F 8 A$ and $F 8 B$ genes, which are both expressed ubiquitously in different tissues [10]. In 2001, F8A gene was shown to code for a $40 \mathrm{kD}$ huntingtin-associated protein, termed HAP4O [12] and is thought to be involved in the aberrant nuclear localization of the huntingtin protein in Huntington disease. The function of $F 8 B$ is not known. Because there is no $F 8 B$ equivalent in the mouse genome, transgenic mice that express the wild-type human $F 8 B$ under the control of a cytomegalovirus promoter have been used to understand its function. Surprisingly, these $F 8 B$ transgenic mice showed growth retardation, microcephaly and severe ocular defects, evidence that should encourage further studies of this protein [13].

\subsection{3-2005: F8 Intron 22 Inversion Discovery and Detection}

In 1993, two research groups-one led by Jane Gitschier in USA and the other one by Francesco Giannelli in UK-independently observed that one half of severe HA patients had no detectable mutation in the promoter, coding sequences or normal RNA processing signals of the $F 8$ gene $[11,14]$. Instead they revealed a unique mRNA defect that prevents the amplification of the message across the boundary between exon 22 and 23. This feature located the defect to internal regions of intron 22 and a model was proposed based on recombination between homologous F8A sequences located in intron 22 and upstream of the $F 8$ gene. Such event of homologous recombination would lead to an inversion of all intervening DNA and a disruption of the $F 8$ gene. Both groups presented evidence to support this model. 
Figure 1. Schematic view of the $F 8$ int $22 h$ normal gene regions (A, B) and int $22 h$-related recombination variants (C-G). From top down, the last $\mathrm{Mb}$ of $\mathrm{Xq} 28$ is shown representing: (A) the normal $F 8$ wild-type variant $h 123$ (according to Xq->Xtel orientation of int $22 h-1$, $h-2$ and $h$-3 sequences); (B) the normal $F 8$ wild-type variant $h 132$ (non-deleterious inversion polymorphism $h 123 / h 132$ ); (C) HA-associated Inv22 type I originating from recombination between $h 1$ and $h 3$ on normal variant $h 123$ shown in (A); (D) HA-associated Inv22 type II originating from recombination between $h 1$ and $h 2$ on normal variant $h 132$ shown in (B); (E) HA-associated Del22 type I originating from recombination between equally oriented int $22 h-1$ and $h-3$ on variant $h 132$ (B); (F) HA-associated Del22 type II originating from recombination between int22h-1 and $h-2$ on variant $h 123$ (A), (Del22 notation: NC_000023.10: g.154,118,607_154,615,713del); and (G) Example of non-HA-associated Dup22 originating from recombination between equally oriented int $22 h-1$ and $h-2$ on variant h123 (A), (Dup22 notation: NC_000023.10: g.154,118,607_154,615,713dup).

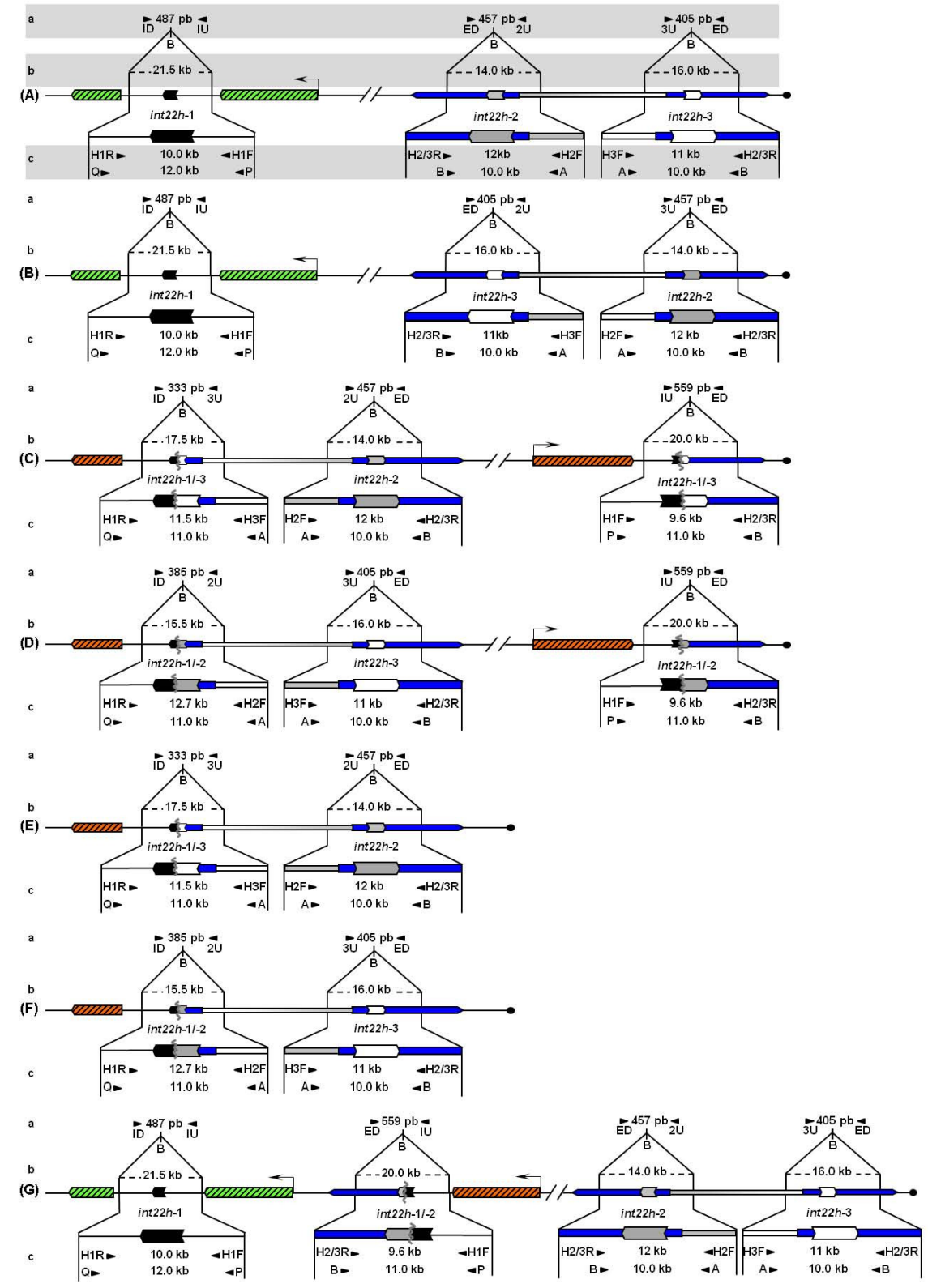


Unbroken $F 8$ gene sequences are shown as green hatched boxes and rearranged $F 8$ sequences as orange hatched boxes; intragenic int $22 h-1$ is shown as a closed chevron; int $22 h-2$ and int $22 h-3$, within the arms of a large imperfect palindrome (blue), are shown as grey and open chevrons, respectively. Chimeric int $22 h$ sequences are denoted as [/] e.g., int22h-1/-2 represents the chimera between $h-1$ and $h$-2. Each schematic displays: (a) ISPCR based approaches developed by Rossetti et al. [15,16], wherein "B" represents a $B c l$ I restriction site after self-end ligation; (b) Southern blot analysis as described by Lakich et al. (1993) [11], wherein dashed lines show Bcl I restriction fragment sizes (kb); and (c) LDPCR based approaches of Bagnall et al. (2006) [17] (upper) and Liu et al. (1998) [18] (lower). Please refer to text for further explanation of details, including derivation of primers with orientation marked by arrowheads.

Lakich et al. (1993) further described a Southern blot assay based on $B c l$ I restriction and an F8A probe for which the sizes of two of the three normal hybridization bands were characteristically altered in patients presenting intron 22 inversions (Inv22) [11] [Figure 1A-D(b)]. They suggested that this assay should permit genetic prediction of HA in approximately $45 \%$ of families with severe disease [11]. Both the USA and UK groups found that this mutation occurred at the surprising rate of approximately $4 \times 10^{-6}$ per gene, per gamete, per generation $[11,14]$.

\subsubsection{First Generation: Southern Blot Analysis as the Gold Standard and Early Findings about Inv22}

Southern blot analysis, as described by Lakich et al. (1993) [11], is still considered the reference method for Inv22 genotyping. These investigators showed that Inv22 can present two different band patterns named distal or type I, and proximal or type II (Inv22-1 and Inv22-2, respectively). Inv22 Southern blot analysis is defined by $B c l$ I enzyme restriction and a labeled probe (900 bp Eco RI-Sac I fragment of plasmid p462.6, ATCC \#57203) corresponding to the F8A gene located within F8 intron 22 and therefore also the two extragenic copies. Accordingly, Southern blot analysis resolves different patterns each containing three signals per allele, i.e., no-Inv22 (normal allele) associated with signals of $21.5,16$, and $14 \mathrm{~kb}$ [Figure $1 \mathrm{~A}(\mathrm{~b})$ and $1 \mathrm{~B}(\mathrm{~b})$ ]; Inv22-1, with signals of 20.0, 17.5 and $14.0 \mathrm{~kb}$ [Figure 1C(b)]; and Inv22-2, with signals of 20.0, 16.0, and 15.5 kb [Figure 1D(b)].

Southern blot analysis is technically robust, enables identification of all types of inversions (Inv22-1 and Inv22-2), and permits a semiquantitative evaluation of Inv22 heterozygous carrier mosaicism as in the case described by Oldenburg et al. (2000) [19]. However, this technique is labor-intensive requiring 8-10 days to obtain the results. Use of hazardous radiochemicals is a further disadvantage and requires authorized personnel, although use of chemiluminescence probe labeling may circumvent these potential risks.

Interestingly, Rossiter et al. (1994) [20] found that Inv22 originates predominantly from male germ cells and hypothesized that the presence of a second X chromosome in female meiosis would hinder the intrachromosomal non-allelic pairing required for Inv22. They presented convincing evidence supporting their findings using linkage analysis. This approach confirmed that, when occurring at the grandparents' generation, the Inv22 was always associated with the grandfather germline (20 out of 20 informative families studied), whereas only one out of 50 mothers of sporadic cases with severe HA and the Inv22 were carriers. Contemporaneously, Tizzano et al. (1995) [21] observed in a Spanish 
population that all mothers of patients with isolated HA caused by the Inv22 resulted from carriers, also indicating that Inv22 originates in male germ cells.

Oldenburg et al. (2000) [19] reported the first instance of Inv22 presenting as somatic mosaicism in a female, affecting only about $50 \%$ of lymphocyte and fibroblast cells. Supposing a postzygotic de novo mutation as the usual cause of somatic mosaicism, the finding implies that the Inv22 mutation is not restricted to meiotic cell divisions but can also occur during mitotic cell divisions, either in germ cell precursors or in somatic cells.

Aiming to define the exact extent of the homologous sequences involved in the Inv22 crossing over event, Naylor et al. (1995) [22] studied an intragenic clone containing $F 8$ intron 22, which contains a copy of $F 8 A$, and two extragenic clones each with a single copy of $F 8 A$ located by the Xq telomere using PCR amplification, chemical cleavage of mismatch (CCM) and DNA sequencing. They precisely defined the repeated region of $9.5 \mathrm{~kb}$ and named it int $22 \mathrm{~h}-1$ (intron 22 homologous region-1) (intragenic to F8), and int22h-2 and int22h-3 (both extragenic to F8). The inversion junctions were shown to represent precise exchanges between the int $22 h$ repeats without insertions or deletions, thus providing conclusive evidence for homologous recombination [22]. The three copies of int $22 h$ were compared along more than $8 \mathrm{~kb}$ of their length, using CCM analysis, and found to be $99.9 \%$ similar [22]. The presence of such long and almost identical inverted repeats near the Xq telomere could account for the high frequency at which the inversions occur [22].

Antonarakis et al. (1995) [23] collected data on 2,093 samples from laboratories all over the world and concluded that the common inversion mutations are found in $42 \%$ of patients with severe HA (35\% of Inv22 type I, $7 \%$ of type II and $0.05 \%$ of rare variants such as types IIIa and IIIb). Whereas $98 \%$ of all mothers of patients with Inv22 were carriers, data from this study was only one de novo inversion event occurring in maternal somatic cells for every 25 mothers of sporadic cases. When the maternal grandparental origin of inversions was examined the ratio of de novo occurrences in male:female germ cells was 69:1. In Argentina, De Brasi et al. (2000) [24] found similar figures for Inv22 type I and type II, although they did not find rare types in a group of 34 patients with severe HA (i.e., $41 \%$ of total Inv22, 35\% of Inv22-1 and 6\% of Inv22-2). According to previous series and the evidence discussed above, the Argentine series showed that all mothers of patients with the Inv22 (and particularly those mothers of isolated cases of hemophilia) were conventional heterozygous carriers, as detected in peripheral blood DNA samples, excluding the possibility of de novo mutation in their gonads.

\subsubsection{Second Generation: Long Distance-PCR Based Approaches}

During the early 1990s, the Inv22 was detectable only by labor-intensive Southern blot analysis. Therefore, a simpler, more rapid and less expensive test for Inv22 genotyping was highly desirable. Steve Sommer in USA designed a single-tube PCR assay that combines overlapping PCR [25] with long distance-PCR (LD-PCR) [26] to achieve the genetic diagnosis of Inv22 causing severe HA [18]. The new method was simple, rapid and relatively inexpensive and thus became the method of choice in many laboratories worldwide.

The inversion was detected by performing LD-PCR directly from genomic DNA with four primers that differentiate the wild-type, Inv22, and carrier genotypes. Two primers, $\mathrm{P}$ and $\mathrm{Q}$, located within the $F 8$ at positions $-1,212$ bp and $+1,334$ bp flanking int $22 h-1$, when combined with two different primers, 
A and B, flanking the two extragenic repeats int $22 h-2$ and int $22 h-3$ each at $-167 \mathrm{bp}$ and $+118 \mathrm{bp}$, yield segments PQ $(12 \mathrm{~kb})$ and $\mathrm{AB}(10 \mathrm{~kb})$ in a hemizygous individual without Inv22 and segments PB $(11 \mathrm{~kb})$ and AQ $(11 \mathrm{~kb})$ along with the $10 \mathrm{~kb}$ AB segment from the intact extragenic homolog in a patient with the Inv22 [Figure 1A-D(c)]. This assay does not differentiate Inv22 types I and II. Inv22 female carriers produce $\mathrm{PQ}, \mathrm{PB}, \mathrm{AQ}$, and $\mathrm{AB}$ segments. In all cases, an $\mathrm{AB}$ segment serves as an internal control because at least one copy of int $22 h-2$ or int $22 h-3$ remains intact. The three long amplimers were separated by agarose gel electrophoresis $0.6 \%$ for $6-8$ hours [18].

Efficient amplification of the four segments depended on three unusual modifications for LD-PCR protocols: (i) high concentrations of dimethyl-sulfoxide; (ii) addition of 7-deaza-dGTP; and (iii) high concentration of a mix of Taq and Pwo DNA polymerases [18]. However, one of the segments was amplified much more efficiently than the others under standard three-temperature cycling conditions. Consequently, to facilitate the uniform amplification of the multiple regions, subcycling-PCR was included in this protocol [27].

The accomplishment to amplify long amplimers encompassing int $22 h$ duplicons by Sommer's group opened the possibility to investigate a highly informative restriction fragment length polymorphism (RFLP) of enzyme Xba I [28]. Notably, the contemporary reports of El-Maari et al. (1999) [29] and De Brasi et al. [30] both described methods based on hemispecific LD-PCR for Xba I RFLP genotyping, one primer targeting single copy DNA on $F 8$ intron 22 and the second primer targeting int $22 h$ repeat sequence. By application of the same approach of hemispecific LD-PCR for int $22 h-1$ specific amplification followed by nested PCR amplification, Bowen et al. (2000) [31] presented a new RFLP of the restriction enzyme Msp I that proved heterozygous in about $46 \%$ of females of Caucasian origin. In addition, De Brasi et al. (2003) [32] reported streamlined genotyping of the Xba I and Msp I RFLP by use of a separate LD-PCR product obtained with primers P and Q [18] to specifically amplify int $22 h-1$ followed by nested PCR. The authors reported a combined heterozygosity of $63 \%$ in Argentine population, which is an exceptionally high figure for such linked markers (750 bp).

Contrasting with the significant virtues of LD-PCR for Inv22 genotyping, amplification of such long amplimers $(>10 \mathrm{~kb})$ including a tract of about $3.3 \mathrm{~kb}$ with $79 \%$ of CG content made this assay somewhat dependent on narrow ranges of input DNA qualities, thermocycling and reagent conditions [27]. With an objective to improve Inv22 genotyping efficiency, Bowen and Kenney (2003) [33] unleashed the multiplex LD-PCR single tube reaction [18] into four separate LD-PCR reactions for each of the primer pairs $\mathrm{PQ}, \mathrm{PB}, \mathrm{AQ}$ and $\mathrm{AB}$ [Figure $1 \mathrm{~A}-\mathrm{D}(\mathrm{c})]$. This separation permitted more robust amplification for each primer pair, and results were readily interpretable using standard agarose gel electrophoresis.

\subsubsection{Third Generation: Inverse Shifting-PCR Based Approaches}

In order to overcome the problems associated with direct amplification of int $22 \mathrm{~h}$ duplicons, Rossetti et al. (2005) [15] designed an alternative approach for Inv22 genotyping based on a variant of the classical inverse-PCR designed by Ochman et al. (1988) [34]. Novel Inv22 inverse-PCR analysis was inspired by the typical signal shift from 21.5 to $20 \mathrm{~kb}$ on Southern blot autoradiograms indicative of the presence of Inv22 type I or type II [14]. This alternative inverse-PCR based protocol included three steps: (i) $B c l$ I restriction; (ii) self-ligation of restriction fragment ends yielding $B c l$ I circles; and (iii) standard multiplex PCR analysis (Figure 2). Three years later, this approach was named inverse 
shifting-PCR (IS-PCR) [16] because it differs from classical inverse-PCR in that primers target at short definite distances from the site of restriction/ligation and, therefore, a sequence change associated with a particular rearrangement generates a chimeric circle that is recognized by a shift in primer usage and is ultimately reflected by the predicted size of IS-PCR product (Figure 2).

Figure 2. Schematic view of the inverse shifting-PCR approach. The simpler version of IS-PCR was described by Rossetti et al. (2005) [15] and involves three steps: (a) genomic DNA is subjected to restriction digestion yielding fragments (in this case $\mathrm{Bcl}$ I fragments), (b) restriction fragments self-end ligation (performed in large volumes), which forms (c) DNA circles that represent templates for a standard multiplex PCR analysis (on background some non relevant circles are shown); (d) PCR products from relevant circles are resolved by conventional electrophoresis. Lane 1 shows wild-type allele-specific products (-), lane 2, a male patient hemizygous for Inv22 (+), lane 3, heterozygous Inv22 carrier female (+/-). On the diagram int $22 h-1$ is shown as a closed chevron; int $22 h-2$ and int $22 h-3$ as grey and open chevrons, respectively. Oligonucleotide primer ID indicates intragenic downstream, IU, intragenic upstream and ED, extragenic downstream.

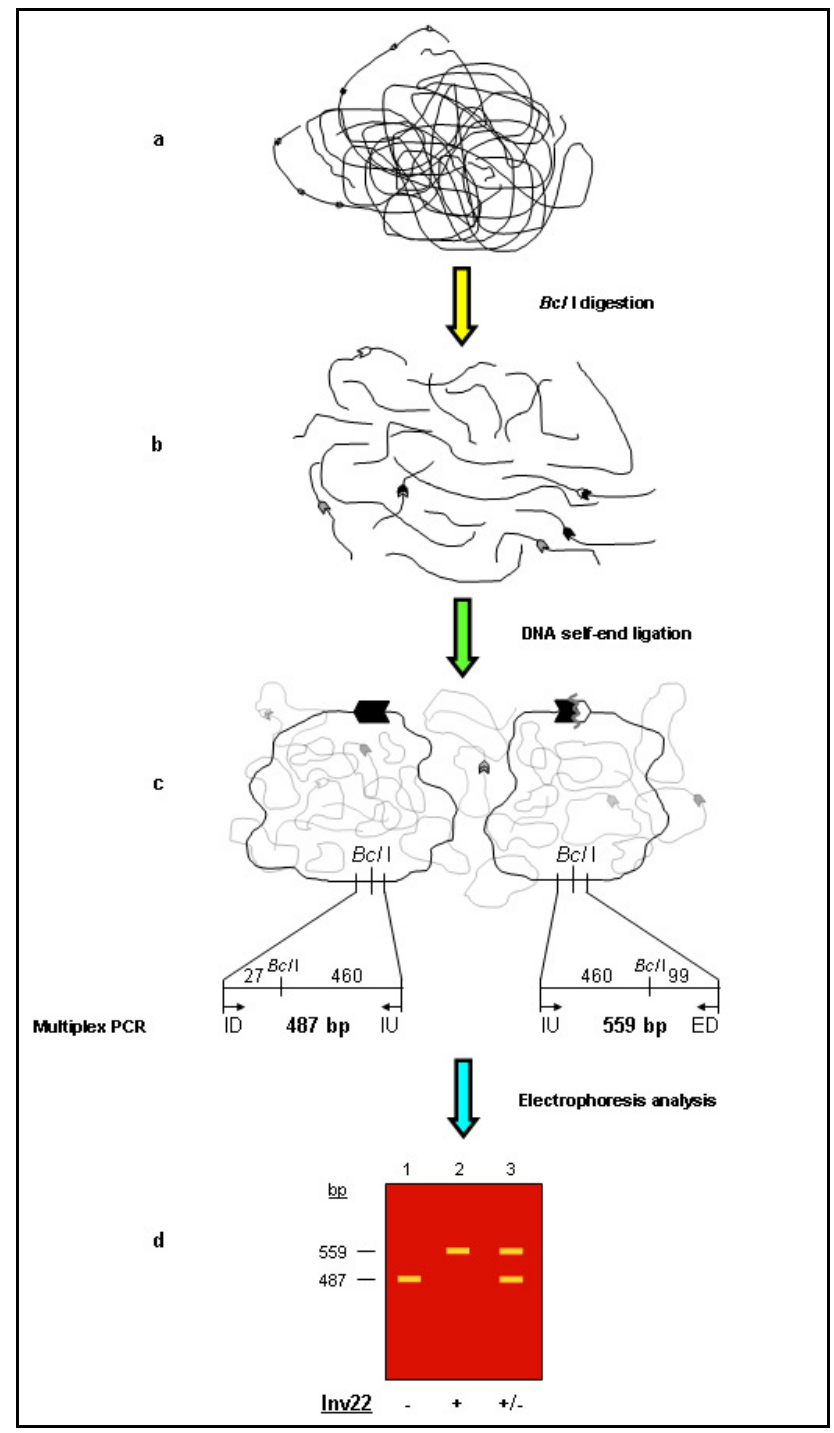


Inv22 analysis by IS-PCR was achieved using three different primers (ID, IU, ED) that yielded a $487 \mathrm{bp}$ amplicon (ID/IU) for the wild-type intragenic allele and a $559 \mathrm{bp}$ amplicon (ED/IU) for the Inv22 allele (Figure 2). PCR products were analyzed by standard agarose gel electrophoresis. It is important to reinforce that primers for IS-PCR were targeted to regions free of human repeats and low-complexity DNA by masking the relevant regions [15].

\subsection{5-2011: Completion of the Human X-Chromosome Sequence and Definition of Hypothetical int22h-Mediated Rearrangements. Unraveling a Complex Picture}

The traditional picture stated by Naylor et al. (1995), which reigned for a decade, proposed that both int $22 h-2$ and int $22 h-3$ should be in opposite orientation to int $22 h-1$ on the X-chromosome [22]. In this scenario, intrachromosomal homologous recombination between int $22 h-1$ and either of the two extragenic copies may result in the two varieties (types) of the recurrent inversions that cause almost half of cases of severe HA. It was believed that int $22 h-1$ interacts with either the proximal (int $22 h-2)$, or the distal (int22h-3) extragenic copy, generating either Inv22 type II or type I, respectively. By this model, interaction between int $22 h-1$ and int $22 h-3$ would be favored over those between int $22 h-1$ and int22h-2, thus explaining their relative frequencies (4:1, Inv22 type I: type II) (Naylor et al. 1995) [22].

Availability of the DNA sequence of the X-chromosome in 2005 showed that int $22 h-2$ and int $22 h-3$ are found within the arms of a large imperfect palindrome, and only int $22 h-3$ should be involved in these inversions [35] (Figure 1A and 1B). The duplicated inverted sections (arms) are $50 \mathrm{~kb}$-long and are separated by $67 \mathrm{~kb}$ of non-duplicated spacer sequence (Figure $1 \mathrm{~A}$ and 1B). The int $22 h-2$ and int $22 h$-3 regions lie adjacent to the spacer sequence, and the more proximal of these (traditionally int $22 h-2)$ is in the same orientation as int $22 h-1$. Therefore, recombination between int $22 h-1$ and int $22 h$-2 should lead to deletions or duplications rather than inversion [36].

Bagnall et al. (2005) suggested an attractive hypothesis to explain the relative frequencies of type I and type II inversions [36]. These investigators proposed that the large palindrome arms could recombine frequently with each other to generate an inversion of the central $67 \mathrm{~kb}$ segment (spacer) that expresses in the human population as a structural inversion polymorphism with frequencies of $80 \%$ and $20 \%$ for the two variants, i.e., $h 123$ and h132, respectively (Figure 1A and 1B).

\subsubsection{More on the Second and Third Generation. New Tests to Allow Comprehensive Detection of int22h-Related Rearrangements}

Unfortunately, Inv22 genotyping by LD-PCR (1998) [18] and IS-PCR (2005) [15] does not permit discrimination of type I and type II Inv22 patterns (Figure 1C and 1D), nor, perhaps more importantly, do these methods allow detection of hypothesized int22h-related deletions (Del22) (Figure 1E and 1F) or duplications (Dup22) (Figure 1G). These limitations of rapid approaches for Inv22 genotyping opened up the possibility that molecular diagnosis may be misrepresented in some cases.

In a bid to overcome these limitations and to improve molecular diagnosis of Inv22, new protocols for Inv22 detection based on LD-PCR (2006) [37] and IS-PCR (2008) [16] were developed with the intention to identify all int $22 \mathrm{~h}$ rearrangements. Both of these revised protocols allow discrimination of Inv22 type I and type II patterns (Figure 1C and 1D), int22h-mediated deletions (Del22-1, Del22-2) 
(Figure 1E and 1F), and duplications (Dup22) (Figure 1G) by using complementary or additional diagnostics tests (Figure 1A-G(a) and $1 \mathrm{~A}-\mathrm{G}(\mathrm{c})$ ).

Bagnall et al. (2006) [37] developed an LD-PCR based method for specific detection of Inv22 patterns type I and type II using a single test with four primers (named H1R, H1F, H2F and H3F) yielding a $10 \mathrm{~kb}$ product in normal DNA representing the intact int $22 \mathrm{~h}$ - 1 region, and signals of 11.5 and $12.7 \mathrm{~kb}$ in DNA from patients with the Inv22 type I and type II, respectively [Figure 1C-D(c)]. These latter signals represent the more centromeric reciprocal of the int $22 h$ recombined sequences, which respectively contain part of int $22 h-3$ and int $22 h-2$. DNA samples from Inv22 heterozygous carriers show one of the mentioned Inv22 specific signals accompanied by the $10 \mathrm{~kb}$ signal seen in normal DNA that contains the non recombined copy of int22h-1 (Figure 1A-B(c)). As equally-oriented int $22 h$-mediated duplications and/or deletions were likely to occur, it is useful to have complementary tests for detecting and distinguishing them from the inversions that cause severe HA. Consequently, Bagnall et al. (2006) [37] designed two complementary tests $\left(\mathrm{Co}^{\circ}\right.$ and $\left.\mathrm{Co} 2^{\circ}\right)$ using two combinations of primers. The test $\mathrm{Co}^{\circ}$ with primers $\mathrm{H} 1 \mathrm{~F}$ and $\mathrm{H} 2 / 3 \mathrm{R}$ shows a signal of $9.6 \mathrm{~kb}$ from samples with the Inv22-1, Inv22-2 and Dup22 [Figure $1 \mathrm{C}(\mathrm{c}), 1 \mathrm{D}(\mathrm{c})$ and $1 \mathrm{G}(\mathrm{c})$ ], whereas Del22 type I or type II associates with an absence of signals; and $\mathrm{Co} 2^{\circ}$ with primers $\mathrm{H} 1 \mathrm{R}, \mathrm{H} 3 \mathrm{~F}$ and $\mathrm{H} 2 \mathrm{~F}$ shows an absence of signals from samples with Dup22, and a signal of $11.5 \mathrm{~kb}$ from either Inv22 or Del 22 type I alleles [Figure $1 \mathrm{C}(\mathrm{c})$ and $1 \mathrm{E}(\mathrm{c})$ ] and $12.7 \mathrm{~kb}$ from either Inv22 or Del 22 type II alleles [Figure $1 \mathrm{D}(\mathrm{c})$ and $1 \mathrm{~F}(\mathrm{c})$ ].

Likewise, Rossetti et al. (2008) [16] modified their earlier reported IS-PCR protocol to resolve all $\mathrm{Bcl}$ I restriction fragments detected by classical Southern blot analysis [11]. This modified protocol enables detection of Inv22 type I and type II as well as Del22 type I and type II, and Dup22 [16]. Similar to its precursor, this modified IS-PCR protocol avoids direct amplification of int $22 h$ duplicons, and uses two standard PCR tests for the same substrate ( $\mathrm{Bcl}$ I circles). The modified protocol includes two multiplex PCR assays: (i) a diagnostic test, which is pattern-sensitive and differentiates HA causative Inv22 and Del22 mutations from non-HA causative Dup22 and normal variants; and (ii) a complementary test intended to distinguish between Inv22 and Del22, and between Dup22 and normal allele. The diagnostic test applies primer ID with a set of three primers $\mathrm{U}$ (IU, 2U and 3U), enabling discrimination of normal/Dup22 allele, associated with a signal of 487 bp [Figure 1A-B(a) and 1G(a)], from Inv22/Del22 type I, with a signal of $333 \mathrm{bp}$ [Figure 1C(a) and 1E(a)], and Inv22/Del22 type II with a signal of $385 \mathrm{bp}$ [Figure $1 \mathrm{D}(\mathrm{a})$ and $1 \mathrm{~F}(\mathrm{a})$ ]. The complementary test applies primer ED with the same set of three primers $U$, and extends diagnostic test findings [16]. On the complementary test the normal allele shows two signals of 457 and 405 bp [Figure 1A-B(a)]; Dup22, three signals of 559, 457 and 405 bp [Figure 1G(a)]; Inv22 type I, two signals of 457 and 559 bp [Figure 1C(a)]; Del22 type I, only one signal of 457 bp [Figure 1E(a)]; Inv22 type II, two signals of 405 and 559 bp [Figure 1D(a)]; and Del22 type II shows only one signal of 405 bp [Figure $1 F(a)$ ].

IS-PCR based approaches for Inv22 genotyping have proved to enable detection and semi-quantitative assessment of carrier mosaicisms, and performed robustly over wide ranges of DNA qualities and procedural conditions, including for prenatal diagnosis [38]. The key step to achieve successful inverse-PCR protocols is the formation of DNA circles from restriction fragments by self-end ligation. Rossetti et al. (2008) [16] estimated the circularization efficiency range from 2-10 units of templates per circle for the formation of DNA circles of approximately $20 \mathrm{~kb}$. 
Despite ongoing efforts to develop technical approaches that would correctly diagnose hypothetical equally-oriented int22h-mediated rearrangements [36] (Figure 1), only recently have such genotypes been found. Notably, a recent paper of Abou-Elew et al. (2011) [39] reported three cases with signal patterns associated with Del22 (two Del22 type II and one Del22 type I) in a group of 13 Egyptian patients with HA by use of the new IS-PCR based two-test approach. This finding is somewhat unexpected taking into account that Del22 involves a loss of more than $500 \mathrm{~kb}$ of genomic DNA spanning a number of genes, five of which predict well characterized syndromes with specifically defined phenotypes in addition to HA [40]. In addition, Abelleyro et al. (2011) [40] presented a different practical approach to support the molecular diagnosis of Del22, by stating the absence or presence of a number of evenly spaced STS (sequence tagged sites) in order to confirm or to exclude the Del22 associated gap, respectively.

\section{Significance of the Human Genome Project for Inv22 Detection and Diagnosis of HA}

This review of an important area of molecular diagnosis in humans clearly shows that the development of new genotyping methods for int $22 h$-mediated rearrangements relies on the extraordinary achievements of the Human Genome Project. In particular, the completion and release of the human X-chromosome sequence [35] permitted an accurate definition of both hypothetical and well established int22h-mediated rearrangements. De Brasi and Bowen (2008) [41] made use of widely available bioinformatic resources, such as BLAST (basic local alignment search tool) [42] and Smith-Waterman [43] algorithms, to calculate the exact extent of int $22 h$ duplicons and their nucleotide sequence differences, the size and location of the large inverted repeats as the arms of the large imperfect palindrome int $22 h-2$ and int $22 h-3$, and to precisely define the full range of int $22 h$-mediated rearrangements (i.e., Inv22, Del22 and Dup22).

Moreover, it has been demonstrated that bioinformatic resources developed from Human Genome Project initiatives, are providing essential tools for the accurate design of experiments in the molecular biology field. The recent design of both LD-PCR and IS-PCR based approaches for genotyping int $22 h$-mediated rearrangements offers two clear examples of this [37,16]. IS-PCR-based genotyping for int22h-related rearrangements was designed using the latest version at that time of the nucleotide sequence of the human X chromosome, GenBank accession NC_000023.9, nt.153,500,000-154,387,415, which encompassed the entire $F 8$, and the centromeric and telomeric arms of the $168 \mathrm{~kb}$ imperfect palindrome in which int $22 h-2$ and int $22 h-3$ are located.

Sequence analysis required extensive application of in silico tools including $B c l$ I restriction mapping, DNA sequence alignments, repeat masking, virtual circle formation by self-end ligation and oligonucleotide primer selection. Additionally, in order to illustrate the usefulness of bioinformatic developments in the area of molecular diagnostic medicine even further, the repeat masker web server (URL: http://www.repeatmasker.org) was used to map human repeats throughout all relevant sequences in order to limit the primer target sites to human repeat-free regions $[15,16]$.

In summary, three genotyping methods presently allow Inv22 analysis to discriminate all int22h-mediated rearrangements (i.e., Inv22, Del22 and Dup22), thus reducing potential diagnostic mistakes to a minimum. These methods include Southern blot analysis by Lakich et al. (1993) [11], the discriminative three-test based LD-PCR by Bagnall et al. (2006) [37], and the discriminative two-test 
based IS-PCR by Rossetti et al. (2008) [16]. These three generations of molecular methods for genotyping the hemophilia inversion hotspot are valuable examples of international cooperation, experimental ingenuity and, ultimately, scientific evolution to solve challenging molecular diagnostic problems.

\section{Acknowledgments}

The following institutions provided support to our research projects: René Barón Foundation, Alberto J. Roemmers Foundation, Florencio Fiorini Foundation; Adolfo H. Aztiria Foundation, National Research Council CONICET, National Agency for Promotion of Science and Technology ANPCyT, Academia Nacional de Medicina and World Federation of Hemophilia.

In addition we thank the hematologists Miguel Candela, Raúl Pérez Bianco and Miguel de Tezanos Pinto for helping us and supporting our research initiatives; and Christiane Dosne Pasqualini for her kind help with English correction throughout the manuscript.

\section{References}

1. Saiki, R.; Gelfand, D.; Stoffel, S.; Scharf, S.; Higuchi, R.; Horn, G.; Mullis, K.; Erlich, H. Primer-directed enzymatic amplification of DNA with a thermostable DNA polymerase. Science 1988, 239, 487-491.

2. Graw, J.; Brackmann, H.H.; Oldenburg, J.; Schneppenheim, R.; Spannagl M.; Schwaab, R. Haemophilia A: From mutation analysis to new therapies. Nat. Rev. Genet. 2005, 6, 488-501.

3. Kasper, C.K.; Buzin, C.H. Genetics of Hemophilia A and B. An Introduction for Clinicians, 2009, 1st ed.; Southland Publications: Pasadena, CA, USA, 2009; pp. 27-29.

4. Kemball-Cook, G.; Tuddenham, E.G.; Wacey, A.L. The factor VIII structure and mutation resource site: HAMSTeRS version 4. Nucleic Acids Res. 1998, 26, 216-219.

5. Oldenburg, J.; Schröder, J.; Hermann Brackmann, H.H.; Müller-Reible, C.; Schwaab, R.; Tuddenham, E. Environmental and genetic factors influencing inhibitor development. Semin. Hematol. 2004, 41, 82-88.

6. Gitschier, J.; Wood, W.I.; Goralka, T.M.; Wion, K.L.; Chen, E.Y.; Eaton, D.H.; Vehar, G.A.; Capon, D.J.; Lawn, R.M. Characterization of the human factor VIII gene. Nature 1984, 312, 326-330.

7. Fujita, P.A.; Rhead, B.; Zweig, A.S.; Hinrichs, A.S.; Karolchik, D.; Cline, M.S.; Goldman, M.; Barber, G.P.; Clawson, H., Coelho, A.; et al. The UCSC genome browser DATABASE: Update 2011. Nucleic Acids Res. 2010, 39, D876-D882.

8. Levinson, B.; Kenwrick, S.; Lakich, D.; Hammonds, G.; Gitschier, J. A transcribed gene in an intron of the human factor VIII gene. Genomics 1990, 7, 1-11.

9. Freije, D.; Schlessinger, D. A 1.6-Mb contig of yeast artificial chromosomes around the human factor VIII gene reveals three regions homologous to probes for the DXS115 locus and two for the DXYS64 locus. Am. J. Hum. Genet. 1992, 51, 66-80.

10. Levinson, B.; Kenwrick, S.; Gamel, P.; Fisher, K.; Gitschier, J. Evidence for a third transcript from the human factor VIII gene. Genomics 1992, 14, 585-589.

11. Lakich, D.; Kazazian, H.H., Jr; Antonarakis, S.E.; Gitschier, J. Inversions disrupting the factor VIII gene are a common cause of severe haemophilia A. Nat. Genet. 1993, 5, 236-241. 
12. Peters, M.F.; Ross, C.A. Isolation of a 40 k-Da Huntingtin-associated protein. J. Biol. Chem. 2001, 276, 3188-3194.

13. Valleix, S.; Jeanny, J.C.; Elsevier, S.; Joshi, R.L.; Fayet, P.; Bucchini, D.; Delpech, M. Expression of human F8B, a gene nested within the coagulation factor VIII gene, produces multiple eye defects and developmental alterations in chimeric and transgenic mice. Hum. Mol. Genet. 1999, 8, 1291-1301.

14. Naylor, J.; Brinke, A.; Hassock, S.; Green, P.M.; Giannelli, F. Characteristic mRNA abnormality found in half the patients with severe haemophilia A is due to large DNA inversions. Hum. Mol. Genet. 1993, 2, 1773-1778.

15. Rossetti, L.C.; Radic, C.P.; Larripa, I.B.; De Brasi, C.D. Genotyping the hemophilia inversion hotspot by use of inverse PCR. Clin. Chem. 2005, 51, 154-158.

16. Rossetti, L.C.; Radic, C.P.; Larripa, I.B.; De Brasi, C.D. Developing a new generation of tests for genotyping hemophilia causative rearrangements involving int $22 \mathrm{~h}$ and int $1 \mathrm{~h}$ hotspots in the factor VIII gene. J. Thromb. Haemost. 2008, 6, 830-836.

17. Bagnall, R.D.; Giannelli, F.; Green, P.M. Int22h-related Inversions causing hemophilia A: A novel insight into their origin and a new more discriminant PCR test for their detection. J. Thromb. Haemost. 2006, 4, 591-598.

18. Liu, Q.; Nozari, G.; Sommer, S.S. Single-tube polymerase chain reaction for rapid diagnosis of the inversion hotspot of mutation in haemophilia A. Blood 1998, 92, 1458-1459. (Erratum in Blood 1999, 93, 2141.)

19. Oldenburg, J.; Rost, S.; El-Maarri, O.; Leuer, M.; Olek, K.; Müller, C.R.; Schwaab, R. De novo factor VIII gene intron 22 inversion in a female carrier presents as a somatic mosaicism. Blood 2000, 96, 2905-2906.

20. Rossiter, J.P.; Young,M.; Kimberland, M.L.; Hutter, P.; Ketterling, R.P.; Gitschier, J.; Horst, J.; Morris, M.A.; Schaid, D.J.; de Moerloose, P. Factor VIII gene inversions causing severe hemophilia A originate almost exclusively in male germ cells. Hum. Mol. Genet. 1994, 3, 1035-1039.

21. Tizzano, E.F.; Domènech, M.; Baiget, M. Inversion of intron 22 in isolated cases of severe hemophilia A. Thromb. Haemost. 1995, 73, 6-9.

22. Naylor, J.A.; Buck, D.; Green, P.; Williamson, H.; Bentley, D.; Giannelli, F. Investigation of the factor VIII intron 22 repeated region (int22h) and the associated inversion junctions. Hum. Mol. Genet. 1995, 4, 1217-1224.

23. Antonarakis, S.E.; Rossiter, J.P.; Young, M.; Horst, J.; de Moerloose, P.; Sommer, S.S.; Ketterling, R.P.; Kazazian, H.H., Jr.; Négrier, C.; Vinciguerra, C.; et al. Factor VIII gene inversions in severe hemophilia A: results of an international consortium study. Blood 1995, 86, 2206-2212.

24. De Brasi, C.D.; Candela, M.; Cermelj, M.; Slavutsk, I.R.; Larripa, I.B.; Pérez Bianco, R.; de Tezanos Pinto, M. Intron 22 factor VIII gene inversions in Argentine families with severe haemophilia A. Haemophilia 2000, 6, 21-22.

25. Liu, Q.; Thorland, E.C.; Heit, J.A.; Sommer, S.S. Overlapping PCR for bidirectional PCR amplification of specific alleles: A rapid one-tube method for simultaneously differentiating homozygotes and heterozygotes. Genome Res. 1997, 7, 389-398.

26. Barnes, W.M. PCR amplification of up to 35-kb DNA with high fidelity and high yield from lambda bacteriophage templates. Proc. Natl. Acad. Sci. USA 1994, 91, 2216-2220. 
27. Liu, Q.; Sommer, S.S. Subcycling-PCR for multiplex long-distance amplification of regions with high and low GC content: Application to the inversion hotspot in the factor VIII gene. Biotechniques 1998, 25, 1022-1028.

28. Wion, K.L.; Tuddenham, E.G.D.; Lawn, R.M. A new polymorphism in the factor VIII gene for prenatal diagnosis of haemophilia A. Nucleic Acids Res. 1986, 14, 4535-4542.

29. El-Maarri, O.; Oldenburg, J.; Cağlayan, S.H. Intron 22-specific long PCR for the Xba I polymorphism in the factor VIII gene. Br. J. Haematol. 1999, 105, 1120-1122.

30. De Brasi, C.D.; Bowen, D.J.; Collins, P.W.; Larripa, I.B. Specific analysis of the intron $22 \mathrm{XbaI}$ polymorphism of the human factor VIII gene using long-distance PCR. Br. J. Haematol. 1999, 107, 566-568.

31. Bowen, D.J.; De Brasi, C.D.; Larripa, I.B.; Collins, P.W. A new polymorphism in the human factor VIII gene: implications for linkage analysis in haemophilia A and for the evolution of int22h sequences. Br. J. Haematol. 2000, 111, 544-548.

32. De Brasi, C.D.; Rossetti, L.C.; Larripa, I.B. Rapid genotyping of XbaI and MspI DNA polymorphisms of the human factor VIII gene: Estimation of their combined heterozygosity in the Argentinean population. Haematologica 2003, 88, 232-234.

33. Bowen, D.J.; Keeney, S. Unleashing the long-distance PCR for detection of the intron 22 inversion of the factor VIII gene in severe haemophilia A. Thromb. Haemost. 2003, 89, 201-202.

34. Ochman, H.; Gerber, A.S.; Hartl, D.L. Genetic applications of an inverse polymerase chain reaction. Genetics 1988, 120, 621-623.

35. Ross, M.T.; Grafham, D.V.; Coffey, A.J.; Scherer, S.; McLay, K.; Muzny, D.; Platzer, M.; Howell, G.R.; Burrows, C.; Bird, C.P.; et al. The DNA sequence of the human X chromosome. Nature 2005, 434, 325-337.

36. Bagnall, R.D.; Giannelli, F.; Green, P.M. Polymorphism and hemophilia A causing inversions in distal Xq28: A complex picture. J. Thromb. Haemost. 2005, 3, 2598-2599.

37. Bagnall, R.D.; Giannelli, F.; Green, P.M. Int22h-related inversions causing hemophilia A: A novel insight into their origin and a new more discriminant PCR test for their detection. J. Thromb. Haemost. 2006, 4, 591-598.

38. Radic, C.P.; Rossetti, L.C.; Zuccoli, J.R.; Abelleyro, M.M.; Larripa, I.B.; De Brasi, C.D. Inverse shifting PCR based prenatal diagnosis of hemophilia-causative inversions involving int $22 \mathrm{~h}$ and int1h hotspots from chorionic villus samples. Prenat. Diagn. 2009, 29, 1183-1185.

39. Abou-Elew, H.; Ahemed, H.; Raslan, H.; Abdelwahab, M.; Hammoud Mokhtar, D.; Arnaout, H. Genotyping of intron 22-related rearrangements of F8 by inverse-shifting PCR in Egyptian hemophilia A patients. Ann. Hematol. 2011, 90, 579-584.

40. Abelleyro, M.M.; Rossetti, L.C.; Radic, C.P.; Candela, M.; Larripa, I.B.; De Brasi, C.D. Are int22h-mediated deletions a common cause of hemophilia? Ann. Hematol. 2011, in press.

41. De Brasi, C.D.; Bowen, D.J. Molecular characteristics of the intron 22 homologs of the coagulation factor VIII gene: an update. J. Thromb. Haemost. 2008, 6, 1822-1824.

42. Altschul, S.F.; Madden, T.L.; Schäffer, A.A.; Zhang, J.; Zhang. Z.; Miller, W.; Lipman, D.J. Gapped BLAST and PSI-BLAST: A new generation of protein database search programs. Nucleic Acids Res. 1997, 25, 3389-3402. 
43. Smith, T.F.; Waterman, M.S. Identification of common molecular subsequences. J. Mol. Biol. 1981, 147, 195-197.

(C) 2011 by the authors; licensee MDPI, Basel, Switzerland. This article is an open access article distributed under the terms and conditions of the Creative Commons Attribution license (http://creativecommons.org/licenses/by/3.0/). 